\title{
Optimal Paths for Avoiding a Radiating Source
}

\author{
L. S. Pachter \\ Department of Mathematics \\ University of California, Berkeley \\ Berkeley, CA 94720 - 3840 \\ M. Pachter \\ Department of Electrical and Computer Engineering \\ Air Force Institute of Technology (AFIT/ENG) \\ Wright-Patterson AFB, OH 45433 - 7765
}

\begin{abstract}
We consider the problem of navigating between points in the plane so as to minimize the exposure to a radiating source. Specifically, given two points $z_{1}, z_{2}$ in the complex plane, we solve the problem of finding the path $C(t)(0 \leq t \leq 1)$ such that $C(0)=z_{1}, C(1)=z_{2}$ and $\int_{0}^{1} \frac{\left|C^{\prime}(t)\right|}{|C(t)|^{k}} d t$ is minimized. The parameter $k$ specializes to a number of interesting cases: in particular $k=2$ pertains to the passive sensor avoidance problem and $k=4$ entails the active radar avoidance problem. The avoidance paths which minimize exposure may have infinite arc-length. To overcome this problem we introduce a weighted exposure and path length optimization problem whose solution requires a variational approach. The optimal trajectory results we obtain are surprisingly intuitive in the cases of interest.
\end{abstract}

\section{Introduction}

The problem of path planning so as to avoid a radiating source can be formalized as follows: Let $S$ and $p$ be points in the plane with $R=|S-p|$. Define the "exposure" relative to $S$ at $p$ to be the quantity $\frac{1}{R^{k}}(k \geq 2)$. The problem is to find a path between two points $A$ and $B$ in the plane so that during a time interval $0 \leq t \leq t_{f}$, a vehicle traveling at constant speed $V=1$ minimizes the cumulative exposure - see, e.g., [1]:

$$
J=\int_{0}^{t} \frac{1}{R^{k}} d t .
$$

It is convenient to choose $S$ to be the origin $O$ and to express the trajectory in polar coordinates $R(\theta), 0 \leq \theta \leq \theta_{f}$, and $\theta_{f}=\angle A O B$. Since in polar coordinates the arc length $d s=$ $\sqrt{\dot{R}^{2}+R^{2}} d \theta$ and $d t=d s / V$, where $V$ is the vehicle's speed, we have the cost functional

$$
J(R(\theta))=\int_{0}^{\theta_{f}} \frac{\sqrt{\dot{R}^{2}+R^{2}}}{R^{k}} d \theta
$$

The variational problem is to find the optimal trajectory $R^{*}(\theta), 0 \leq \theta \leq \theta_{f}$, which minimizes the functional (1), subject to the boundary conditions

$$
\begin{aligned}
R(0) & =R_{0} \\
R\left(\theta_{f}\right) & =R_{f}
\end{aligned}
$$

Without loss of generality we assume $R_{0} \leq R_{f}$. Thus, the problem parameters are $1 \leq \frac{R_{f}}{R_{0}}$ and $0 \leq \theta_{f} \leq \pi$.

Practical examples of the general problem described above include $k=2$ in which case the exposure corresponds to the radiation from a source in $R^{3}$ where the radiation is expanding outwards on the surface of a sphere. Alternatively, the exposure could relate to the inverse effect of a signal emitted by the moving object 
being received by a passive sensor. In the case $k=4$ the exposure is determined by the strength of a radar signal after being emitted from $S$, bouncing off of $p$ and returning to $S$ (an active radar sensor).

The paper is organized as follows. In Section 2 a geometrical solution of the optimal path for the exposure minimization problem is obtained using Möbius transformations [2]. In Section 3 a variational approach, which also applies to the more general class of weighted exposure and path length minimization problems, is presented, followed by concluding remarks in Section 4 .

\section{Geometrical approach}

The optimization problem of minimizing the functional (1) can be approached geometrically by applying the theory of Möbius transformations [2]. This approach has the advantage of avoiding the use of the calculus of variations, and provides insight into the geometry of the optimizing paths.

We will work in the setting of the extended complex plane $C_{\infty}$ which is just the complex plane together with a point at infinity. Since we are in the complex plane, the points $A, B$ become $z_{1}, z_{2} \in C_{\infty}$, namely points in the extended complex plane. In this language the problem becomes to find a path $C(t)$ such that $C(0)=z_{1}$, $C(1)=z_{2}$ and $\int_{0}^{1} \frac{\left|C^{\prime}(t)\right| d t}{|C(t)|^{k}}$ is minimized.

\subsection{Finding the optimal path}

Let $f(z)$ be the transformation $f(z)=\frac{-1}{(k-1) z^{k-1}}$ and let $\gamma(t)=f(C(t))$ be the image of $C(t)$ under the function $f(z)$. The arc length of $\gamma(t)$ from $f\left(z_{1}\right)$ to $f\left(z_{2}\right)$ is given by

$$
\begin{aligned}
\int_{0}^{1}\left|\gamma^{\prime}(t)\right| d t & =\int_{0}^{1}\left|\left[\frac{1}{C(t)^{k-1}}\right]^{\prime}\right| d t \\
& =\int_{0}^{1}\left|\frac{C^{\prime}(t)}{C^{k}(t)}\right| d t \\
& =\int_{0}^{1} \frac{\left|C^{\prime}(t)\right| d t}{|C(t)|^{k}}
\end{aligned}
$$

which is exactly the total exposure to the source as we move from $z_{1}$ to $z_{2}$.
It is clear that $\int_{0}^{1}\left|\gamma^{\prime}(t)\right| d t$ is minimized when $\gamma(t)=t z_{1}+(1-t) z_{2}$ is a straight line. It follows that the exposure between $z_{1}$ and $z_{2}$ is minimized when $C(t)$ is the image under $f^{-1}(z)$ (here the -1 indicates the inverse of $f$ ) of a straight line between $f\left(z_{1}\right)$ and $f\left(z_{2}\right)$.

The case where $k=2$ is particularly interesting because then $f(z)$ is a special case of a Möbius transformation $M(z)=\frac{a z+b}{c z+d}$ where we have $a=0, b=-1, c=1, d=0$. The special transformation $f(z)$ is also known as an inversion. Möbius transformations have a number of important properties which we summarize below:

- $M$ is conformal

- The image under $M$ of any circle is a circle. In particular, the image under $M$ of a circle containing the origin is a line together with the point at infinity (we consider this to be a circle).

- The collection of Möbius transformations form a group.

The inverse of the function $f(z)=\frac{-1}{z}$ in the group of transformations is just itself $(f)$ is idempotent!) and so it follows that the exposure between $z_{1}$ and $z_{2}$ is minimized when $C(t)$ is the arc of the circle determined by $0, z_{1}, z_{2}$ that avoids the origin and contains as its endpoints $z_{1}$ and $z_{2}$ ! The exposure is given by

$$
\int_{0}^{1} \frac{\left|C^{\prime}(t)\right| d t}{|C(t)|^{2}}=\left|f\left(z_{1}\right)-f\left(z_{2}\right)\right|=\left|\frac{1}{z_{1}}-\frac{1}{z_{2}}\right| .
$$

This result is summarized in

Theorem 1. The optimal trajectory $R^{*}(\theta)$ which connects points $A$ and $B$ at a distance $R_{0}$ and $R_{f}$ from the passive sensor located at the origin $O$, and minimizes the exposure to the sensor according to the cost functional

$$
J(R(\theta))=\int_{0}^{\theta_{f}} \frac{\sqrt{\dot{R}^{2}+R^{2}}}{R^{2}} d \theta
$$

is

$$
\frac{R^{*}(\theta)}{R_{0}}=\frac{\sin (\theta+\phi)}{\sin \phi}, 0 \leq \theta \leq \theta_{f}
$$


where $\theta_{f}$ is the angle $\angle A O B$ and $0<\theta_{f}<\pi$. The angle

$$
\phi=\operatorname{Atan}\left(\frac{\sin \theta_{f}}{\frac{R_{f}}{R_{0}}-\cos \theta_{f}}\right)
$$

Moreover, this trajectory is the arc on the cord $A B$ of the circle which circumscribes $\triangle O A B$.

In summary, for the passive sensor avoidance problem the "solution triangle" is $\triangle O A B$, and

- To calculate the optimal trajectory one needs the angle $\phi=\angle A B O$.

- The angle of departure of the optimal trajectory is $\angle A B O=\phi$.

- The angle of arrival of the optimal trajectory is $\angle B A O$.

- The change of heading incurred flying an optimal trajectory is $2 \theta_{f}$.

- The optimal path length is $l^{*}=\frac{\theta_{f}}{\sin \phi} R_{0}$.

The situation for $k=2$ is depicted in Figure 1: The minimizing path is the arc on the cord $A B$ of the circumscribing circle of triangle $A O B$.

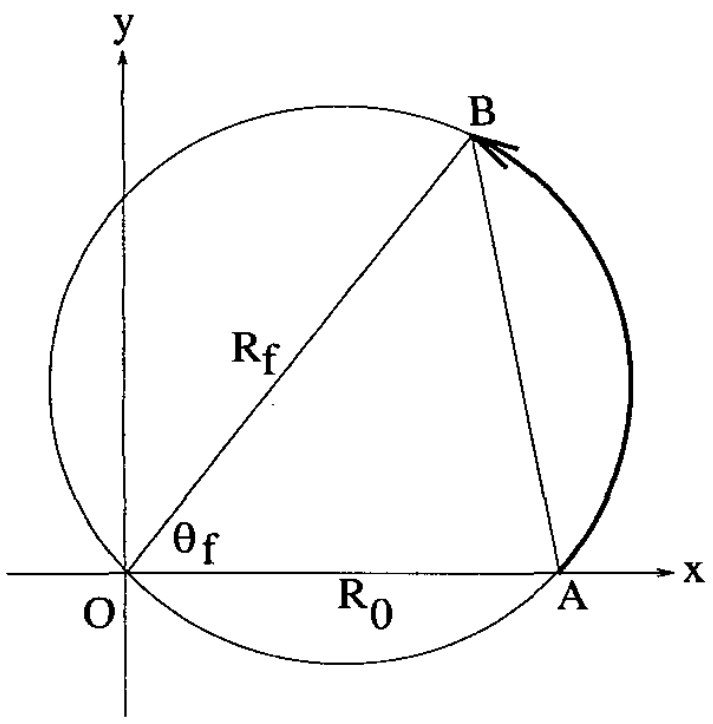

Figure 1: Construction of the optimal trajectory
When $\theta_{f}=0$, the optimal trajectory is the segment $\overrightarrow{A B}$ and the optimal (minimum) cost is

$$
J^{*}=\frac{1}{R_{0}}-\frac{1}{R_{f}} .
$$

When $\theta_{f}=\pi$, the optimal trajectory has an infinite length, however the optimal cost is finite and

$$
J^{*} \rightarrow \frac{1}{R_{0}}+\frac{1}{R_{f}}
$$

It is thus clear that when the angle $\theta_{f}$ is large (close to $\pi$ ), although the existence of an optimal path is guaranteed and the optimal cost is bounded, the length of the optimal path increases without bound.

Another interesting case is $k=4$. In this case the function $f(z)=\frac{-1}{3 z^{3}}$ and the inverse function $f^{-1}(z)=\frac{-1}{(3 z)^{\frac{1}{3}}}$. The exposure is given by

$\int_{0}^{1} \frac{\left|C^{t}(t)\right| d t}{|C(t)|^{4}}=\left|f\left(z_{1}\right)-f\left(z_{2}\right)\right|=\left|\frac{1}{3 z_{1}^{3}}-\frac{1}{3 z_{2}^{3}}\right|$.

and the path $C$ between two points is just

$f^{-1}\left(t \frac{-1}{3 z_{1}^{3}}+(1-t) \frac{-1}{3 z_{2}^{3}}\right)=\frac{-1}{\left(3\left(t \frac{-1}{3 z_{1}^{3}}+(1-t) \frac{-1}{3 z_{2}^{3}}\right)\right)^{\frac{1}{3}}}$.

Thus, the optimal radar avoidance trajectory is an arc of the "rose" function

$$
\frac{R^{*}(\theta)}{R_{0}}=\left[\frac{\sin (\theta+\phi)}{\sin \phi}\right]^{\frac{1}{3}}, 0 \leq \theta \leq \theta_{f},
$$

where the angle

$$
\phi=\operatorname{Atan}\left(\frac{\sin 3 \theta_{f}}{\left(\frac{R_{f}}{R_{0}}\right)^{3}-\cos 3 \theta_{f}}\right)
$$

It is interesting to compare eqs. (6), (10) and eqs. (7), (11) for $k=2$ and $k=4$, respectively.

Finally, it is interesting to note that when $k$ is odd the function $f^{-1}(z)=\frac{i}{((k-1) z)^{\frac{1}{k-1}}}$, and when $k$ is even $f^{-1}(z)=\frac{-1}{((k-1) z)^{\frac{1}{k-1}}}$. 


\subsection{Geometry}

The geometry of minimal paths avoiding a source is particularly interesting in the case $k=2$ as described above. In that case, a "straight line" between two points is the arc of the circle between them, where the circle is determined by the points $A, B$, and the origin $O$. Three points now lie on a straight line if together with the origin they lie on a circle.

We can define a convex region $R$ as usual to be a subset of $C_{\infty}$ with the property that the "line" determined by two points lies in $R$. The convex hull of $n$ points is the intersection of all the convex regions containing the points.

Convex regions are simply the image of convex regions in Euclidean space under the function $f(z)$. Using this idea it is not difficult to see that there are two distinct cases for the convex hull of three points:

1. The Euclidean convex hull of the three points contains the origin: In this case the convex hull consists of the unbounded region outside the circular segments connecting the points.

2. The Euclidean convex hull of the three points does not contain the origin: In this case the convex hull consists of the bounded region inside the circular segments connecting the points.

The convex hull of three points is illustrated in Figure 2. In the second case, the origin is contained in the Euclidean convex hull of the points, so the convex hull in the "avoidance geometry" is the region exterior to the bold arcs.

Also of interest is the shape of the open ball centered at $z_{0}$. That is,

$$
\begin{gathered}
B\left(z_{0}\right)=\left\{z: \int_{0}^{1} \frac{\left|C^{\prime}(t)\right| d t}{|C(t)|^{2}} \leq 1,\right. \\
\left.C(0)=z_{0}, C(1)=z\right\} .
\end{gathered}
$$

This set is clearly the preimage of an open ball in Euclidean plane, namely the preimage of a disk. Since Möbius transformations map circles to circles, and $f^{-1}(z)$ is still a Möbius transformation, the open ball $B\left(z_{0}\right)$ is just a disk (although not necessarily centered at $z_{0}$ ).

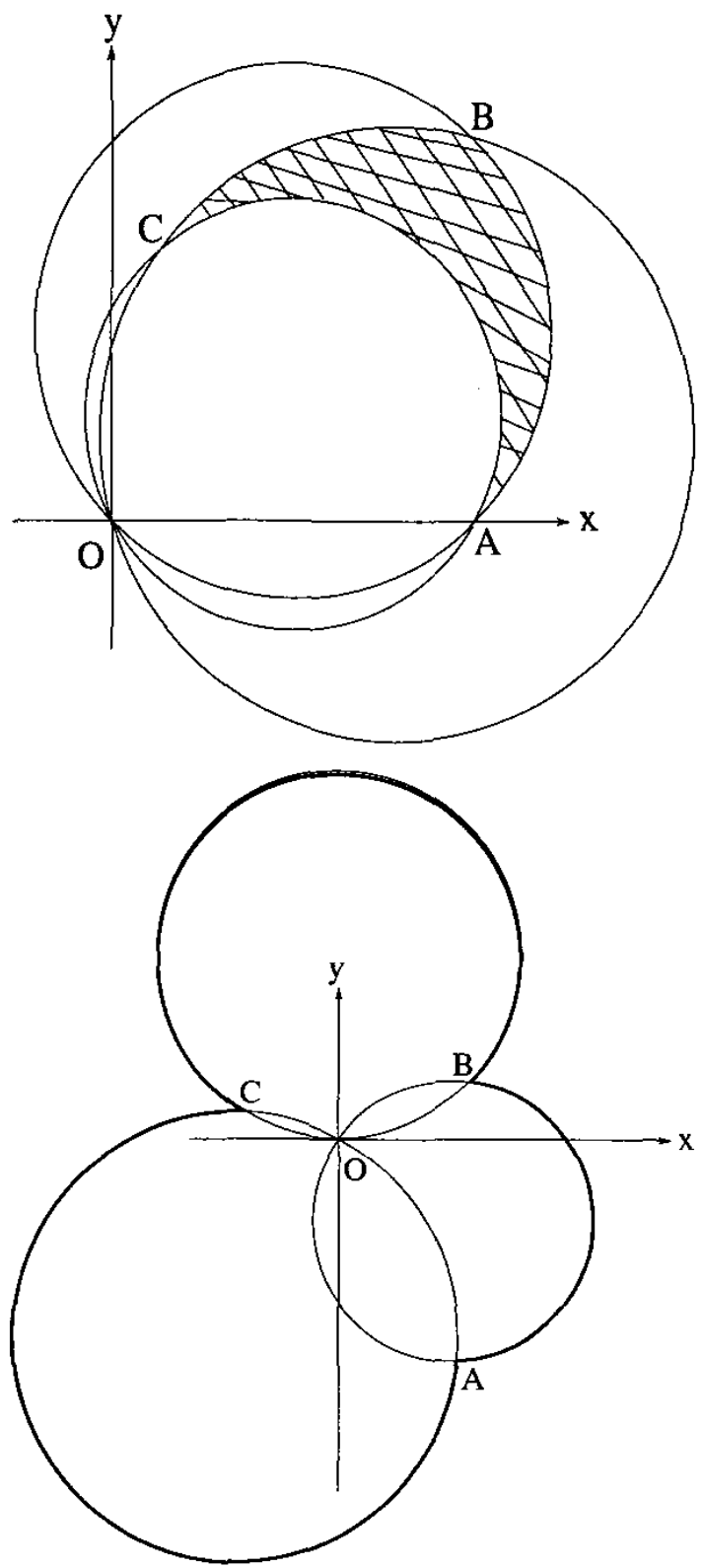

Figure 2: Convex hull of three points

It is not hard to see that in general, the geometry of minimal avoiding paths can be completely understood by inverting straight lines in the Euclidean plane. 
Additional optimization problems amenable to solution using the method of inversion: The initial and/or terminal points, $A$ and $B$, respectively, are replaced by manifolds. Thus, the following additional optimal trajectory problems are of interest.

1) The initial and/or terminal manifold is a straight line.

2) The initial and/or terminal manifold is a circle. Moreover the optimization might require the target circle to be approached from the outside or from the inside.

3) The initial (terminal) manifold is a straight line and the terminal (initial) manifold is a circle.

A particularly interesting case arises when both the initial and terminal manifolds are straigh lines which meet at $O$. In this case an optimal solution does not exist, viz., the length of the optimal trajectory $l^{*} \rightarrow \infty$ and the optimal cost $J^{*} \rightarrow 0$.

When applied to these problems, the classical variational method - see, e.g., Sect. 3 in the sequel - requires us to a) Recognize that, as in the case with specified initial and terminal ponts $A$ and $B$, the optimal trajectory is an arc of a circle which circumscribes $\triangle O A^{\prime} B^{\prime}$, where the yet to be determined trajectory end points $A^{\prime}$ and $B^{\prime}$ lie on the initial and terminal manifolds, respectively, and, b) Evoke the applicable transversality conditions, which in our case require the optimal trajectory to orthogonally approach the initial and terminal manifolds. Thus, using a) and b) reduces the solution of the optimal trajectory problems to geometric construction problems. The geometric method of inversion using the Möbius transformation directly yields the optimal trajectories. Moreover, the non-existence of an optimal solution is directly characterized by the use of a point at infinity in the extended complex plane. However, we will see in the next section that a natural generalization of the problem formulated above so that an optimal solution always exists, does not yield to the technique of Möbius inversion and requires the calculus of variations. Still, the method of inversion does allow one to obtain a lower bound for the minimal cost.

\section{Minimizing a weighted sum of exposure and path length}

In the previous section we defined "exposure" as $\int_{0}^{1} \frac{1}{|S-p|^{k}} d t$. An unsatisfactory property of this definition is that the trajectory which minimizes the exposure between two points might have infinite path length. For example, consider the case $k=2$ where $z_{1}, z_{2}$ and the origin lie on a true straight line with the origin between $z_{1}$ and $z_{2}\left(\theta_{f}=\pi\right)$. In this case the minimal exposure path between $z_{1}$ and $z_{2}$ uses the point at infinity, which makes sense mathematically but is useless for a practical application where we might want to physically move from $z_{1}$ to $z_{2}$ in a finite amount of time. Indeed, this example shows that the exposure might be finite but the path length infinite. If however we move from $z_{1}$ to $z_{2}$ using the shortest Euclidean path, then we will have infinite exposure. A similar situation arises in the case $k=4$ for $\frac{\pi}{3} \leq \theta_{f}$. This leads us to the following optimization problem:

Find a path $C(t)$ such that $C(0)=z_{1}, C(1)=$ $z_{2}$ and $\alpha \int_{0}^{1} \frac{\left|C^{\prime}(t)\right| d t}{|C(t)|^{k}}+\beta \int_{0}^{1}\left|C^{\prime}(t)\right| d t$ is minimized, where $\alpha, \beta$ are positive constants with $\alpha+\beta=1$. By tuning the parameters $\alpha$ and $\beta$ (or, $\lambda \equiv \frac{\beta}{\alpha}$, $\lambda>0$ ), we can give more weight to minimizing the exposure, or to minimizing the path length. The cost functional (5) is now modified to include a weighted sum of the exposure and the path length. Specifically, the cost functional is

$J(R(\theta))=\int_{0}^{\theta_{f}}\left(\frac{\sqrt{\dot{R}^{2}+R^{2}}}{R^{2}}+\lambda \frac{1}{R_{0}^{2}} \sqrt{\dot{R}^{2}+R^{2}}\right) d \theta$ where the weight $0 \leq \lambda$ is used instead of $\alpha$ and $\beta$. Hence, the path length is penalized and the optimal solution entails a tradeoff between exposure and path length. We'll assume $\lambda>0$; when the weight $\lambda=0$ we are back to the optimization problem discussed in Sections 1 and 2. We also nondimensionalize $R$, that is we set $R \rightarrow \frac{R}{R_{0}}$, and similarly, $R_{f} \rightarrow \frac{R_{f}}{R_{0}}$. The boundary conditions (2) and (3) become $R(0)=1$ and $R\left(\theta_{f}\right)=R_{f}\left(\equiv \frac{R_{f}}{R_{0}}\right)$, respectively, and the cost 
functional is

$J(R(\theta))=\int_{0}^{\theta_{f}}\left(\frac{\sqrt{\dot{R}^{2}+R^{2}}}{R^{2}}+\lambda \sqrt{\dot{R}^{2}+R^{2}}\right) d \theta$

We minimize the cost functional (13) subject to the boundary conditions (2) and (3).

This problem cannot be solved with the technique used in Section 2 and we must apply the calculus of variations [3]. Closed-form solutions for a weighted sum of exposure and path length optimization can be obtained using elliptic integrals [4]. The special case $R_{f}=1$ and $\lambda=1$ is interesting. It can be shown that the following holds.

Theorem 2 The solution of the optimization problem (13) with the path length penalty weight of $\lambda=1$, the boundary condition $\frac{R_{f}}{R_{0}}=1$, and $\forall 0 \leq \theta_{f} \leq \pi$, is a circular arc centered at $O$.

This result is particularly interesting, because although the circular arc centered at $O$ is an extremal in the original exposure minimization problem with the cost functional (5), it is not the optimal trajectory - see, e.g., Remark 1. The optimal trajectory is in fact given by the circular arc shown in Fig. 1. Hence, it is remarkable that the very same circular arc is the optimal solution when the path length is penalized according to eq. (13), and the penalty weight $\lambda=1$.

\section{Conclusions}

The problem of navigating between way points so as to minimize the exposure to a radiating source has been solved. A closed form solution using the Möbius transformation is obtained. The geometry of the optimal paths is most interesting. Also, intersting connections between complex analysis, geometry, and the calculus of variations are established. The variational problem where a weighted sum of exposure and path length is minimized is also considered.

\section{References}

[1] John E. Hershey et al, "Strategic Route Planning and Sensor Fusion", IEEE Trans. on Aerospace and Electronic Systems, Vol. 29 , No. 4, pp. 1137 - 1139, October 1993.

[2] H. Schwerdtfeger, Geometry of Complex Numbers: Circle Geometry, Moebius Transformation, Non-Euclidean Geometry, Dover, 1979.

[3] I. M. Gelfand and S. V. Fomin, Calculus of Variations, Prentice Hall, 1963.

[4] P. F. Byrd and M. D. Friedman, Handbook of Elliptic Integrals for Engineers and Physicists, Springer-Verlag, 1954. 